\title{
High Uric Acid Level Predicts Early Neurological Deterioration in Intracerebral Hemorrhage
}

\begin{abstract}
Xiuqun Gong, $\mathbb{D}^{1, *}$ Zeyu Lu, ${ }^{2, *}$ Xiwu Feng, ${ }^{3, *}$ Kang Yuan, ${ }^{4}$ Mei Zhang,' Xiaosi Cheng,' Min Xue,' Liang Yu,' Jun Lu, (iD) Chuanqing $\mathrm{Yu}^{\prime}$

'Department of Neurology, First Affiliated Hospital of Anhui University of Science and Technology, First People's Hospital of Huainan, Huainan, People's Republic of China; ${ }^{2}$ College of Medicine, Anhui University of Science and Technology, Huainan, People's Republic of China; ${ }^{3}$ Department of Cardiothoracic Surgery, First Affliated Hospital of Anhui University of Science and Technology, First People's Hospital of Huainan, Huainan, People's Republic of China; ${ }^{4}$ Department of Neurology, Jinling Hospital, Nanjing Medical University, Nanjing, People's Republic of China; ${ }^{5}$ Department of Medical Laboratory, College of Medicine, Anhui University of Science and Technology, Huainan, People's Republic of China; ${ }^{6}$ Department of Clinical Laboratory, First Affiliated Hospital of Anhui University of Science and Technology, First People's Hospital of Huainan, Huainan, People's Republic of China

*These authors contributed equally to this work
\end{abstract}

Correspondence: Jun Lu

Department of Medical Laboratory, College of Medicine, Anhui University of Science and Technology, 168 Taifeng Road, Huainan, 23200I, Anhui Province, People's Republic of China

Tel + 86- 13855453 I49

Email cfdxlujun@।26.com

Chuanqing Yu

Department of Neurology, First Affiliated Hospital of Anhui University of Science and Technology, First People's Hospital of Huainan, 203 Huaibin Road, Huainan, 232007, Anhui Province, People's Republic of China

Tel +86- I8949669880

Email yuchuanqin1967@163.com
Objective: Increased level of serum uric acid (UA) is often considered a risk factor for ischemic stroke. However, there are limited data on the association between UA and intracerebral hemorrhage (ICH). This study aimed to examine the connection between UA and early neurological deterioration (END) in patients with ICH.

Methods: This is a prospective observational study. Patients with ICH were enrolled from January 2017 to December 2020. END was diagnosed as the Canadian Stroke Scale (CSS) score decreased $\geq 1$ points between admission and 48 hours. UA was measured at admission. Multivariable logistic regression analysis was performed to explore the relationship between serum UA and END.

Results: Of the 498 enrolled patients, 132 (26.5\%) were developed with END. Patients with END had a significantly higher level of serum UA (332 vs $270 \mu \mathrm{mol} / \mathrm{L}, P<0.001)$. Univariate logistic regression analysis indicated that patients with the highest quartile of UA level had an OR of 3.256 (95\% CI: $1.849-5.734, P<0.001)$ for END compared with those with the lowest quartile of UA level. After adjusting for major confounders, the highest UA quartile remained as an independent predictor for END (OR $=2.282,95 \% \mathrm{CI}: 1.112-$ 4.685, $P=0.013)$.

Conclusion: Higher serum UA level was independently associated with END in patients with $\mathrm{ICH}$; therefore, intervention to lower UA level may be worth considering.

Keywords: early neurological deterioration, intracerebral hemorrhage, uric acid

\section{Introduction}

Intracerebral hemorrhage (ICH) accounts for $10-30 \%$ of all strokes and is the leading cause of stroke-related death and disability. ${ }^{1,2}$ There is compelling evidence from preclinical and clinical research that ICH outcome is strongly affected by a multitude of variables associated with metabolism, ${ }^{3,4}$ inflammation $^{5,6}$ and drug actions. $^{7}$ All these factors may act at the site of cerebral damage and/or at systemic level, and hence, influence neurovascular recovery, secondary-induced damage and systemic complications. The early stage of ICH is extremely unstable, and about $20 \%$ of patients are prone to occur early neurological deterioration (END) within two days of onset, ${ }^{8}$ which is associated with poor prognosis. ${ }^{9}$ Accordingly, it is important to identify risk factors of END to improve clinical outcomes.

Uric acid (UA) is the final product of purine metabolism in the body. It has been proven that hyperuricemia is related to gout, chronic kidney disease, hypertension, diabetes, coronary heart disease and ischemic stroke. ${ }^{10-16}$ In addition, hyperuricemia potentially leads to poor outcome, increased symptomatic ICH and mortality in ischemic stroke. ${ }^{17-19}$ 
Based on the neurologically damaging effect of hyperuricemia, we speculate that it may play a part in END in patients with ICH. However, the association between UA and END in patients with ICH has not been assessed to date. Therefore, this study aimed to explore the relationship between serum UA levels and END in patients with ICH.

\section{Materials and Methods}

\section{Study Population}

Patients with ICH were enrolled consecutively from the First Affiliated Hospital of Anhui University of Science and Technology from January 2017 to December 2020 in this prospective observational study. Patients were included if they: (1) were diagnosed with ICH verified by CT scans within $24 \mathrm{~h}$ from symptom onset; (2) aged $\geq 18$ years; (3) Glasgow Coma Scale (GCS) score $\geq 9$. The patients were excluded if they: (1) had secondary hemorrhage as a result of tumor, trauma, vascular malformation, aneurysm, hemorrhagic transformation of cerebral infarct and blood coagulation abnormalities; (2) did not re-examine brain CT within 48 hours; (3) underwent intracranial hematoma removal or cerebrospinal fluid drainage within 48 hours; (4) had severe heart, renal or liver diseases. This study was conducted in accordance with the Declaration of Helsinki and approved by the ethics committee of the First Affiliated Hospital of Anhui University of Science and Technology. All participants signed an informed consent form.

\section{Clinical Data}

Demographic characteristics, medical history and clinical variables were all collected from medical records. Stroke severity was assessed using the Canadian Stroke Scale (CSS) score. ${ }^{20}$ The extent of consciousness was determined by the GCS score. Fasting blood samples were collected in the next morning for UA, other biochemical indicators and routine blood tests. Serum UA level was measured by uric acid oxidase reagent on a Dax analyzer (Bayer-Technicon). Hematoma expansion was defined as an increase in ICH volume on follow-up CT scans of either $6 \mathrm{~mL}$ or $>33 \%{ }^{9}$ END was diagnosed as the CSS score decreased $\geq 1$ points between admission and 48 hours. ${ }^{8}$

\section{Statistical Analysis}

Continuous variables were presented as mean $\pm \mathrm{SD}$ or median (interquartile range). Categorical variables were presented as numbers (percentages). Independent $t$ test, Mann-Whitney $U$-test, one-way ANOVA or Kruskal-Wallis H-test as appropriate were employed for continuous variables. Chisquare test or Fisher's exact test was used for categorical data. Multivariate logistic regression analysis was used to explore the relationship between categorical serum UA levels and END. Model 1 was adjusted for age and sex. Model 2 was further adjusted for variables with $P<0.1$ in univariate analysis. Results were expressed as odds ratios (ORs) with 95\% confidence interval (CI). The pattern and magnitude of associations between $\mathrm{UA}$ and END was evaluated using a restricted cubic spline with 4 knots (at 5th, 35th, 65th, 95th) adjusted for covariates as in model 2. Results were considered as statistically significant if two-sided $P<0.05$. The R software package version 4.0 (R Foundation, Vienna, Austria) was utilized for restricted cubic spline test, while SPSS 22.0 (IBM, New York, USA) was adopted for other statistical analyses.

\section{Results}

From January 2017 to December 2020, a total of 632 patients diagnosed with ICH were admitted to the Department of Neurology in the First Affiliated Hospital of Anhui University of Science and Technology, of them, 134 were excluded according to the exclusion criteria: secondary hemorrhage $(\mathrm{n}=28)$, did not re-examine brain CT within 48 hours $(\mathrm{n}=37)$, underwent intracranial hematoma removal or cerebrospinal fluid drainage within 48 hours $(n=38)$ or severe heart, renal or liver diseases $(n=31)$. Finally, 498 patients were enrolled in our analysis. Most patients were men $(64.7 \%)$. The median age of the enrolled patients was 66 (54-76) years. END occurred in 132 patients (26.5\%).

Table 1 illustrates the patients' demographic characteristics, clinical data and laboratory data according to the presence or absence of END. Compared with subjects without END, those with END had higher proportions of concurrent ventricular hemorrhage $(P=0.012)$ and hematoma expansion $(P=0.005)$, higher baseline hematoma volume $(P<0.001)$, baseline GCS score $(P<0.001)$ and CSS score $(P=0.003)$, higher levels of homocysteine $(P=$ $0.030)$, creatinine $(P=0.007)$ and UA $(P<0.001)$.

Table 2 shows that the ascending quantiles of UA was associated with male sex $(P<0.001)$, hypertension $(P<$ $0.001)$, smokers $(P=0.033)$, alcohol drinkers $(P<0.001)$, previous use of antihypertensive drugs $(P=0.018)$, higher rates of concurrent ventricular hemorrhage $(P=0.041)$ and hematoma expansion $(P=0.030)$, higher baseline hematoma $(P=0.033)$, higher systolic and diastolic blood pressure (both $P<0.001$ ), higher prevalence of END $(P<0.001)$, higher levels of homocysteine $(P=0.008)$, triglycerides $(P<0.001)$, blood urea nitrogen $(P<0.001)$ 
Table I Baseline Characteristics of Participants with or without END

\begin{tabular}{|c|c|c|c|c|}
\hline Variables & Total $(n=498)$ & With END $(n=132)$ & Without END $(n=366)$ & $P$ value \\
\hline Age, years & $66.0(54.0-76.0)$ & $65.5(54.0-76.0)$ & $66.0(54.8-76.0)$ & 0.796 \\
\hline Gender, Male, n (\%) & $322(64.7)$ & $92(69.7)$ & $230(62.8)$ & 0.158 \\
\hline \multicolumn{5}{|l|}{ Medical history, n (\%) } \\
\hline Hypertension & 388 (77.9) & $106(80.3)$ & $282(77.0)$ & 0.440 \\
\hline Diabetes & $106(21.3)$ & $28(21.2)$ & $78(21.3)$ & 0.981 \\
\hline Hyperlipidemia & $150(30.1)$ & $42(31.8)$ & $108(29.5)$ & 0.620 \\
\hline Coronary heart disease & $39(7.8)$ & II (8.3) & $28(7.7)$ & 0.802 \\
\hline Atrial fibrillation & $16(3.2)$ & $2(1.5)$ & $14(3.8)$ & 0.197 \\
\hline Prior stroke & $152(30.5)$ & $43(32.6)$ & $109(29.8)$ & 0.550 \\
\hline Smoking & $127(25.5)$ & $37(28.0)$ & $90(24.6)$ & 0.437 \\
\hline Drinking & $114(22.9)$ & $33(25.0)$ & $81(22.1)$ & 0.501 \\
\hline \multicolumn{5}{|l|}{ Medication history, n (\%) } \\
\hline Antihypertensive & $221(44.4)$ & $66(50.0)$ & $155(42.3)$ & 0.129 \\
\hline Antiplatelet & $105(21.1)$ & $34(25.8)$ & $71(19.4)$ & 0.125 \\
\hline Anticoagulant & $3(0.6)$ & $0(0.0)$ & $3(0.8)$ & 0.569 \\
\hline Statin & $88(17.7)$ & $29(22.0)$ & $59(16.1)$ & 0.131 \\
\hline \multicolumn{5}{|l|}{ Hematoma location, n (\%) } \\
\hline Lobe & $54(10.8)$ & $16(12.1)$ & $38(10.4)$ & 0.582 \\
\hline Basal ganglia & $274(55.0)$ & $73(55.3)$ & $201(54.9)$ & 0.939 \\
\hline Thalamus & $114(22.9)$ & $34(25.8)$ & $80(21.9)$ & 0.361 \\
\hline Cerebellum & $39(7.8)$ & $6(4.5)$ & $33(9.0)$ & 0.101 \\
\hline Brainstem & $17(3.4)$ & $4(3.0)$ & $13(3.6)$ & 1.000 \\
\hline Concurrent ventricular hemorrhage, $n$ (\%) & $112(22.5)$ & $40(30.3)$ & $72(19.7)$ & 0.012 \\
\hline Baseline hematoma volume, $\mathrm{mL}$ & $12(8-18)$ & $15(|2-2|)$ & II (7-16) & $<0.001$ \\
\hline Hematoma expansion, $n$ (\%) & 79 (15.9) & $31(23.5)$ & $48(13.1)$ & 0.005 \\
\hline \multicolumn{5}{|l|}{ Clinical characteristics } \\
\hline Body temperature, ${ }^{\circ} \mathrm{C}$ & $36.6(36.4-36.8)$ & $36.6(36.5-36.8)$ & $36.5(36.3-36.8)$ & 0.243 \\
\hline Time from onset to admission, $\mathrm{h}$ & $3.5(2.0-6.0)$ & $3.0(2.0-7.8)$ & $3.5(2.0-6.0)$ & 0.918 \\
\hline Time from onset to first $\mathrm{CT}, \mathrm{h}$ & $3.4(2.3-6.4)$ & $3.4(2.4-7.2)$ & $3.5(2.3-5.5)$ & 0.837 \\
\hline Baseline SBP, mmHg & $165(150-183)$ & $170(150-190)$ & $164(149-180)$ & 0.189 \\
\hline Baseline DBP, mmHg & $100(88-110)$ & $100(90-110)$ & $100(86-110)$ & 0.104 \\
\hline Baseline GCS score & $15(14-15)$ & $15(14-15)$ & $15(15-15)$ & $<0.001$ \\
\hline Baseline CSS score & $7.0(5.0-8.0)$ & $6.5(5.0-7.5)$ & $7.0(5.0-8.0)$ & 0.003 \\
\hline \multicolumn{5}{|l|}{ Laboratory data } \\
\hline Leukocyte, $\times 10^{9} / \mathrm{L}$ & $7.48(5.92-9.34)$ & $7.92(6.54-9.46)$ & $7.39(5.69-9.30)$ & 0.087 \\
\hline Neutrophil, $\times 10^{9} / \mathrm{L}$ & $5.45(4.00-7.32)$ & $5.94(4.56-7.28)$ & $5.34(3.89-7.39)$ & 0.068 \\
\hline Hemoglobin, g/L & $129(119-142)$ & $131(120-143)$ & $129(119-142)$ & 0.235 \\
\hline C-reactive protein, $\mathrm{mg} / \mathrm{L}$ & $2.0(0.9-4.0)$ & $2.0(1.0-4.0)$ & $2.0(0.9-4.9)$ & 0.503 \\
\hline Plasma fibrinogen, $g / L$ & $2.80(2.35-3.31)$ & $2.77(2.33-3.16)$ & $2.82(2.39-3.35)$ & 0.235 \\
\hline Homocysteine, $\mu \mathrm{mol} / \mathrm{L}$ & $12.4(9.0-16.5)$ & $14.1(10.0-18.2)$ & $12.2(8.8-16.0)$ & 0.030 \\
\hline Total cholesterol, $\mathrm{mmol} / \mathrm{L}$ & $4.1(3.5-4.9)$ & $4.1(3.3-5.0)$ & $4.1(3.5-4.8)$ & 0.931 \\
\hline Triglyceride, mmol/L & $1.3(0.9-1.8)$ & $1.3(1.0-2.1)$ & $1.3(0.9-1.7)$ & 0.134 \\
\hline $\mathrm{HDL}, \mathrm{mg} / \mathrm{dL}$ & $1.2(0.9-1.4)$ & $1.2(1.0-1.4)$ & $1.2(0.9-1.4)$ & 0.965 \\
\hline $\mathrm{LDL}, \mathrm{mg} / \mathrm{dL}$ & $2.6(2.1-3.1)$ & $2.6(2.0-3.0)$ & $2.5(2.1-3.1)$ & 0.920 \\
\hline Serum glucose, mmol/L & $5.5(4.8-6.7)$ & $5.6(4.8-6.9)$ & $5.5(4.8-6.7)$ & 0.607 \\
\hline Blood urea nitrogen, $\mathrm{mmol} / \mathrm{L}$ & $4.8(3.9-5.8)$ & $4.8(4.0-5.9)$ & $4.8(3.9-5.7)$ & 0.503 \\
\hline Creatinine, $\mu \mathrm{mol} / \mathrm{L}$ & $88(76-98)$ & $91(77-105)$ & $86(76-96)$ & 0.007 \\
\hline $\mathrm{UA}, \mu \mathrm{mol} / \mathrm{L}$ & $281(224-349)$ & $332(245-408)$ & $270(220-328)$ & $<0.001$ \\
\hline
\end{tabular}

Abbreviations: CSS, Canadian Stroke Scale; DBP, diastolic blood pressure; END, early neurological deterioration; GCS, Glasgow Coma Scale; HDL, high-density lipoprotein; LDL, low-density lipoprotein; SBP, systolic blood pressure; UA, uric acid. 
Table 2 Baseline Characteristics of Participants According to UA Quartiles

\begin{tabular}{|c|c|c|c|c|c|}
\hline \multirow[t]{2}{*}{ Variables } & \multicolumn{4}{|c|}{ UA ( $\mu \mathrm{mol} / \mathrm{L})$} & \multirow[t]{2}{*}{$P$ value for Trend } \\
\hline & QI $(\leq 224, n=126)$ & Q2 (224-28|, n=|26) & Q3 $(281-349, n=125)$ & Q4 $(>349, n=121)$ & \\
\hline Age, years & $67.0(60.0-77.0)$ & $70.0(55.8-79.0)$ & $63.0(52.5-72.5)$ & $64.0(51.0-72.0)$ & 0.001 \\
\hline Gender, Male, $\mathrm{n}(\%)$ & $52(41.3)$ & $81(64.3)$ & $84(67.2)$ & $105(86.8)$ & $<0.001$ \\
\hline \multicolumn{6}{|l|}{ Medical history, n (\%) } \\
\hline Hypertension & $82(65.1)$ & $106(84.1)$ & $96(76.8)$ & $104(86.0)$ & $<0.001$ \\
\hline Diabetes & $35(27.8)$ & $25(19.8)$ & $26(20.8)$ & $20(16.5)$ & 0.173 \\
\hline Hyperlipidemia & $29(23.0)$ & $37(29.4)$ & $42(33.6)$ & $42(34.7)$ & 0.173 \\
\hline Coronary heart disease & $10(7.9)$ & $10(7.9)$ & $10(8.0)$ & $9(7.4)$ & 0.998 \\
\hline Atrial fibrillation & $4(3.2)$ & $6(4.8)$ & $2(1.6)$ & $4(3.3)$ & 0.568 \\
\hline Prior stroke & $35(27.8)$ & $45(35.7)$ & $37(29.6)$ & $35(28.9)$ & 0.523 \\
\hline Smoking & $22(17.5)$ & $32(25.4)$ & $32(25.6)$ & $41(33.9)$ & 0.033 \\
\hline Drinking & $17(13.5)$ & $21(16.7)$ & $31(24.8)$ & $45(37.2)$ & $<0.001$ \\
\hline \multicolumn{6}{|l|}{ Medication history, n (\%) } \\
\hline Antihypertensive & $44(34.9)$ & $53(42.1)$ & $58(46.4)$ & $66(54.5)$ & 0.018 \\
\hline Antiplatelet & $26(20.6)$ & $30(23.8)$ & $28(22.4)$ & $21(17.4)$ & 0.633 \\
\hline Anticoagulant & I (0.8) & $0(0.0)$ & I (0.8) & I (0.8) & 0.796 \\
\hline Statin & $17(13.5)$ & $21(16.7)$ & $25(20.0)$ & $25(20.7)$ & 0.422 \\
\hline \multicolumn{6}{|l|}{ Hematoma location, n (\%) } \\
\hline Lobe & $14(11.1)$ & $11(8.7)$ & $18(14.4)$ & $11(9.1)$ & 0.456 \\
\hline Basal ganglia & $71(56.3)$ & $75(59.5)$ & $62(49.6)$ & $66(54.5)$ & 0.454 \\
\hline Thalamus & $28(22.2)$ & $25(19.8)$ & $29(23.2)$ & $32(26.4)$ & 0.666 \\
\hline Cerebellum & $9(7.1)$ & $14(11.1)$ & $9(7.2)$ & $7(5.8)$ & 0.435 \\
\hline Brainstem & $4(3.2)$ & I (0.8) & $7(5.6)$ & $5(4.1)$ & 0.200 \\
\hline Concurrent ventricular hemorrhage, $n$ (\%) & $21(16.7)$ & $26(20.6)$ & $27(21.6)$ & $38(31.4)$ & 0.041 \\
\hline Baseline hematoma volume, $\mathrm{mL}$ & $10(7-15)$ & $13(8-17)$ & $12(8-18)$ & $13(10-20)$ & 0.033 \\
\hline Hematoma expansion, n (\%) & II (8.7) & $18(14.3)$ & $24(19.2)$ & $26(21.5)$ & 0.030 \\
\hline END, n (\%) & $25(19.8)$ & $24(19.0)$ & $29(23.2)$ & $54(44.6)$ & $<0.001$ \\
\hline \multicolumn{6}{|l|}{ Clinical characteristics } \\
\hline Body temperature, ${ }^{\circ} \mathrm{C}$ & $36.6(36.4-36.8)$ & $36.6(36.4-36.8)$ & $36.7(36.4-36.8)$ & $36.5(36.3-36.7)$ & 0.261 \\
\hline Time from onset to admission, $\mathrm{h}$ & $3.5(2.0-6.0)$ & $3.0(2.0-7.8)$ & & $3.5(2.0-6.0)$ & 0.487 \\
\hline Time from onset to first $\mathrm{CT}, \mathrm{h}$ & $3.0(2.0-7.3)$ & $4.0(2.8-7.0)$ & $4.0(2.0-6.0)$ & $3.0(2.0-6.0)$ & 0.705 \\
\hline Baseline SBP, mmHg & $160(142-175)$ & $167(150-185)$ & $161(145-180)$ & $172(152-192)$ & $<0.001$ \\
\hline Baseline DBP, $\mathrm{mmHg}$ & $95(80-105)$ & $100(85-108)$ & $100(90-110)$ & $100(94-116)$ & $<0.001$ \\
\hline Baseline GCS score & $15(14-15)$ & $15(15-15)$ & $15(15-15)$ & $15(14-15)$ & 0.065 \\
\hline Baseline CSS score & $6.0(4.5-8.0)$ & $7.0(5.0-8.0)$ & $7.0(6.0-8.0)$ & $7.0(6.0-8.0)$ & 0.137 \\
\hline \multicolumn{6}{|l|}{ Laboratory data } \\
\hline Leukocyte, $\times 10^{9} / \mathrm{L}$ & $7.20(5.62-9.16)$ & $8.00(5.71-9.60)$ & $7.40(5.90-9.22)$ & $7.73(6.59-9.49)$ & 0.223 \\
\hline Neutrophil, $\times 10^{9} / \mathrm{L}$ & $5.38(3.73-7.31)$ & $5.68(4.05-7.76)$ & $5.26(3.94-7.11)$ & $5.56(4.16-7.05)$ & 0.467 \\
\hline Hemoglobin, g/L & $126(\mid 16-142)$ & $129(120-142)$ & $132(\mid 19-142)$ & $132(120-142)$ & 0.244 \\
\hline C-reactive protein, $\mathrm{mg} / \mathrm{L}$ & $2.0(1.0-6.0)$ & $2.0(0.9-5.1)$ & $1.7(0.5-4.0)$ & $2.0(1.0-3.0)$ & 0.830 \\
\hline Plasma fibrinogen, $g / L$ & $2.80(2.47-3.39)$ & $2.87(2.42-3.33)$ & $2.73(2.13-3.22)$ & $2.81(2.39-3.29)$ & 0.247 \\
\hline Homocysteine, $\mu \mathrm{mol} / \mathrm{L}$ & $11.3(8.6-14.7)$ & $12.1(9.0-16.9)$ & $12.7(10.3-16.0)$ & $14.4(10.0-18.7)$ & 0.008 \\
\hline Total cholesterol, mmol/L & $4.0(3.4-4.7)$ & $4.1(3.5-5.1)$ & $4.1(3.5-4.8)$ & $4.3(3.6-5.0)$ & 0.237 \\
\hline Triglyceride, $\mathrm{mmol} / \mathrm{L}$ & $1.0(0.8-1.5)$ & $1.3(0.9-1.8)$ & $1.3(1.0-2.0)$ & $1.4(1.0-1.9)$ & $<0.001$ \\
\hline $\mathrm{HDL}, \mathrm{mg} / \mathrm{dL}$ & $1.2(1.0-1.4)$ & $1.2(1.0-1.4)$ & $1.2(1.0-1.3)$ & I.I (0.9-1.4) & 0.309 \\
\hline $\mathrm{LDL}, \mathrm{mg} / \mathrm{dL}$ & $2.5(2.0-3.0)$ & $2.5(2.0-3.1)$ & $2.5(2.0-3.0)$ & $2.7(2.1-3.2)$ & 0.194 \\
\hline Serum glucose, mmol/L & $5.5(4.7-7.6)$ & $5.7(4.9-7.2)$ & $5.4(4.8-6.5)$ & $5.4(4.7-6.3)$ & 0.213 \\
\hline Blood urea nitrogen, $\mathrm{mmol} / \mathrm{L}$ & $4.4(3.5-5.0)$ & $4.7(3.8-5.5)$ & $4.9(3.9-6.0)$ & $5.4(4.6-6.9)$ & $<0.001$ \\
\hline Creatinine, $\mu \mathrm{mol} / \mathrm{L}$ & 77 (69-89) & $86(76-95)$ & $88(80-98)$ & $97(88-118)$ & $<0.001$ \\
\hline
\end{tabular}

Abbreviations: CSS, Canadian Stroke Scale; DBP, diastolic blood pressure; END, early neurological deterioration; GCS, Glasgow Coma Scale; HDL, high-density lipoprotein; LDL, low-density lipoprotein; SBP, systolic blood pressure; UA, uric acid. 
Table 3 Logistic Regression Model of UA and END

\begin{tabular}{|c|c|c|c|c|c|}
\hline & \multicolumn{4}{|c|}{ Quartiles of UA ( $\mu \mathrm{mol} / \mathrm{L})$} & \multirow[t]{2}{*}{$P$ value } \\
\hline & $\begin{array}{c}\text { QI }(\leq 224, \\
n=126)\end{array}$ & $\begin{array}{c}\text { Q2 (224-28I, } \\
n=\mid 26)\end{array}$ & $\begin{array}{c}\text { Q3 }(28 \mid-349, \\
n=125)\end{array}$ & $\begin{array}{c}\text { Q4 (>349, } \\
n=121)\end{array}$ & \\
\hline Unadjusted model & Reference & $0.95 \mid(0.509-1.774)$ & $1.220(0.667-2.231)$ & $3.256(1.849-5.734)$ & $<0.001$ \\
\hline Model I & Reference & 0.951 (0.505-I.792) & $1.233(0.664-2.288)$ & $3.303(1.795-6.079)$ & $<0.001$ \\
\hline Model 2 & Reference & $0.823(0.419-1.614)$ & $\mathrm{I} .043(0.530-2.05 \mathrm{I})$ & $2.282(1.112-4.685)$ & 0.013 \\
\hline
\end{tabular}

Notes: Model I: adjusted for age and sex; model 2: further adjusted for variables with $P$ value $<0.1$ in univariate analysis (concurrent ventricular hemorrhage, baseline hematoma volume, baseline GCS score, baseline CSS score, leukocyte, neutrophil, homocysteine and creatinine).

Abbreviations: CSS, Canadian Stroke Scale; END, early neurological deterioration; GCS, Glasgow Coma Scale; UA, uric acid.

and creatinine $(P<0.001)$. Also, patients with higher UA were younger $(P=0.001)$.

Table 3 exhibits the results of the binary logistic regression of END. In the unadjusted model, compared with the first quartile, patients with UA levels in the fourth quartile, were more likely to have END (OR 3.256, 95\% CI 1.849-5.734, $P<0.001)$. The association remained significant after adjusting for the potential confounders (OR 2.282, 95\% CI 1.112-4.685, $P=0.013$ ).

Multiple-adjusted restricted cubic spline regression showed an ascending trend of UA $(P=0.100$ for nonlinearity, as shown in Figure 1) with the risk of END.

\section{Discussion}

This study reveals a significantly increased risk of END among ICH individuals with higher serum UA levels after adjusting for a series of potential confounders. To the best of our knowledge, this is the first study to investigate the association between UA and END in patients with ICH.

The results of previous studies on risk factors of END showed discrepancy, mainly related to the differences in diagnostic criteria and research objects. It has been reported that END was defined as a decrease in the GCS score of $\geq 3$ or death within the first 72 hours, ${ }^{21}>2$ points increase in the NIHSS score within a 72-hour period, ${ }^{22}$ or a decrease in the CSS score of $\geq 1$ points within 48 hours. ${ }^{8}$ In view of the high reliability and validity of CSS score for the assessment of neurological impairment in patients with stroke, ${ }^{23}$ and the relatively convenient scoring procedures, END was defined as the CSS score decreased $\geq 1$ points between admission and 48 hours in the current research. ${ }^{8}$ In this prospective study, END occurred in $26.5 \%$ of patients with ICH, slightly higher

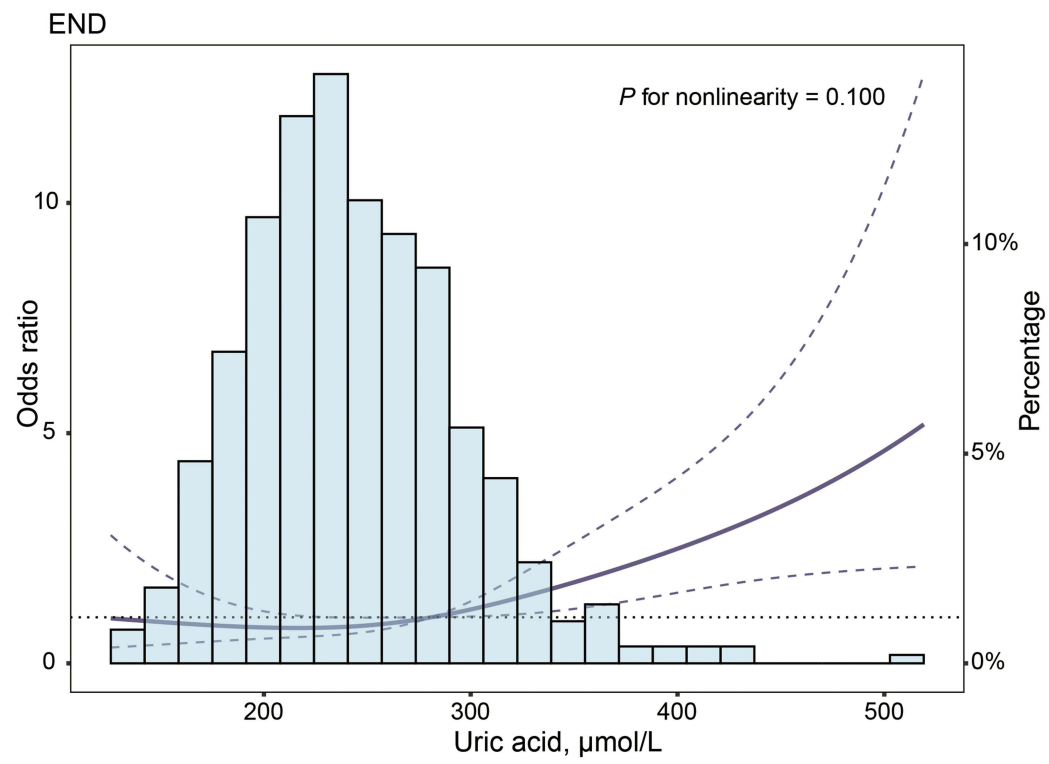

Figure I Association of UA with risk of END. Odds ratio and $95 \% \mathrm{Cl}$ were derived from restricted cubic spline regression, with knots placed at 5 th, 35 th, 65 th, and 95 th percentiles of UA. Odds ratio was adjusted for the same variables as in model 2 (Table 3). The solid line represented the odds ratio and the dashed lines represented the $95 \%$ confidence interval.

Abbreviations: $\mathrm{Cl}$, confidence interval; END, early neurological deterioration; UA, uric acid. 
than the previous study, which may be due to ethnic differences in the study population. ${ }^{8}$

Although the exact mechanisms between UA and END in patients with ICH remained unclear, the following might explain it. First, previous studies have shown that a higher white blood cell count is predictive of END in patients with $\mathrm{ICH}$, suggesting that inflammatory response may be involved in END. ${ }^{8,21}$ Serum UA, on the other hand, promotes the release of a range of inflammatory mediators, such as neutrophils count, C-reactive protein, interleukin-1 $\beta$ (IL-1 $\beta$ ), IL-6, IL-18, and tumor necrosis factor-a (TNF-a), ${ }^{24,25}$ which may in turn lead to END. Secondly, serum UA has pro-oxidant properties by increasing the production of reactive oxygen species (ROS). ${ }^{26}$ Then, the increased oxidative stress level can aggravate secondary brain injury after ICH through inflammatory response, apoptosis, autophagy and destruction of blood-brain barrier, ${ }^{27}$ and eventually contribute to END. Last but not the least, individuals with higher levels of UA were more likely to have larger baseline hematoma volume, higher proportions of intraventricular hemorrhage and hematoma expansion, and all of which have been shown to be risk factors associated with END in patients with $\mathrm{ICH}^{8}{ }^{8,9}$

Some limitations should be noted in this study. First, this was a single-center study with a relatively small sample size, thereby limiting the ability to extend the finding. Secondly, the UA level was detected only once on admission, but not dynamically monitored during the study. The pattern of dynamic change of UA could provide better prognostic information. Thirdly, recent emerging lines of evidence suggest that high blood pressure variations are important predictors of the prognosis of $\mathrm{ICH}^{28,29}$ However, our study did not take this factor into account. Finally, we had little background information, such as purine consumption, history of gout and exercise habit that would affect admission serum UA concentration.

\section{Conclusions}

In conclusion, an elevated serum UA level was independently associated with END in patients with ICH. Therefore, intervention to lower UA level may be worth considering.

\section{Acknowledgments}

This work was partly supported by the National Innovation and Entrepreneurship Training Program for College Students (202010361110) and Huainan Guidance Science and Technology Plan Project (2020104).

\section{Disclosure}

All authors declare that there were no conflicts of interest.

\section{References}

1. van Asch CJ, Luitse MJ, Rinkel GJ, et al. Incidence, case fatality, and functional outcome of intracerebral haemorrhage over time, according to age, sex, and ethnic origin: a systematic review and meta-analysis. Lancet Neurol. 2010;9:167-176. doi:10.1016/S14744422(09)70340-0

2. Feigin VL, Lawes CM, Bennett DA, et al. Stroke epidemiology: a review of population-based studies of incidence, prevalence, and case-fatality in the late 20th century. Lancet Neurol. 2003;2(1):43-53. doi:10.1016/S1474-4422(03)00266-7

3. Zarean E, Lattanzi S, Looha MA, et al. Glycemic gap predicts in-hospital mortality in diabetic patients with intracerebral hemorrhage. $J$ Stroke Cerebrovasc Dis. 2021;30(5):105669. doi:10.1016/j.jstrokecerebrovasdis.2021.105669

4. Jafari M, Di Napoli M, Lattanzi S, et al. Serum magnesium level and hematoma expansion in patients with intracerebral hemorrhage. J Neurol Sci. 2019;398:39-44. doi:10.1016/j.jns.2019.01.027

5. Di Napoli M, Slevin M, Popa-Wagner A, et al. Monomeric c-reactive protein and cerebral hemorrhage: from bench to bedside. Front Immunol. 2018;9:1921. doi:10.3389/fimmu.2018.01921

6. Lattanzi S, Cagnetti C, Provinciali L, et al. Neutrophil-to-lymphocyte ratio and neurological deterioration following acute cerebral hemorrhage. Oncotarget. 2017;8:57489-57494. doi:10.18632/ oncotarget. 15423

7. Mahmoudi J, Majdi A, Lattanzi S, et al. Imidazoline receptor agonists for managing hypertension may hold promise for treatment of intracerebral hemorrhage. Curr Mol Med. 2018;18:241-251. doi:10.2174/ 1566524018666180926163712

8. Godoy DA, Boccio A. Early neurologic deterioration in intracerebral hemorrhage: predictors and associated factors. Neurology. 2005;64:931-932; author reply 931-932. doi:10.1212/WNL.64.5.931-a

9. Lord AS, Gilmore E, Choi HA, et al. Time course and predictors of neurological deterioration after intracerebral hemorrhage. Stroke. 2015;46:647-652. doi:10.1161/STROKEAHA.114.007704

10. Zhang Q, Gong H, Lin C, et al. The prevalence of gout and hyperuricemia in middle-aged and elderly people in Tibet autonomous region, china: a preliminary study. Medicine (Baltimore). 2020;99 (2):e18542. doi:10.1097/MD.0000000000018542

11. Li L, Yang C, Zhao Y, et al. Is hyperuricemia an independent risk factor for new-onset chronic kidney disease?: a systematic review and meta-analysis based on observational cohort studies. $B M C$ Nephrol. 2014;15:122. doi:10.1186/1471-2369-15-122

12. Kuwabara M, Hisatome I, Niwa K, et al. Uric acid is a strong risk marker for developing hypertension from prehypertension: a 5-year Japanese cohort study. Hypertension. 2018;71:78-86. doi:10.1161/ HYPERTENSIONAHA.117.10370

13. Sundström J, Sullivan L, D'Agostino RB, et al. Relations of serum uric acid to longitudinal blood pressure tracking and hypertension incidence. Hypertension. 2005;45:28-33. doi:10.1161/01. HYP.0000150784.92944.9a

14. Lytvyn Y, Perkins BA, Cherney DZ. Uric acid as a biomarker and a therapeutic target in diabetes. Can J Diabetes. 2015;39:239-246. doi:10.1016/j.jcjd.2014.10.013

15. Yang Y, Tian J, Zeng C, et al. Relationship between hyperuricemia and risk of coronary heart disease in a middle-aged and elderly Chinese population. J Int Med Res. 2017;45:254-260. doi:10.1177/ 0300060516673923

16. Zhang S, Liu L, Huang YQ, et al. The association between serum uric acid levels and ischemic stroke in essential hypertension patients. Postgrad Med. 2020;132:551-558. doi:10.1080/00325481.20 20.1757924

17. Weir CJ, Muir SW, Walters MR, et al. Serum urate as an independent predictor of poor outcome and future vascular events after acute stroke. Stroke. 2003;34:1951-1956. doi:10.1161/01.STR.0000 081983.34771.D2 
18. Yuan K, Zhang X, Chen J, et al. Uric acid level and risk of symptomatic intracranial haemorrhage in ischaemic stroke treated with endovascular treatment. Eur J Neurol. 2020;27:1048-1055. doi:10.1111/ene. 14202

19. Storhaug HM, Norvik JV, Toft I, et al. Uric acid is a risk factor for ischemic stroke and all-cause mortality in the general population: a gender specific analysis from the troms $ø$ study. BMC Cardiovasc Disord. 2013;13(1):115. doi:10.1186/1471-2261-13-115

20. Côté R, Battista RN, Wolfson C, et al. The Canadian neurological scale: validation and reliability assessment. Neurology. 1989;39:638-643. doi:10.1212/WNL.39.5.638

21. Sun W, Peacock A, Becker J, et al. Correlation of leukocytosis with early neurological deterioration following supratentorial intracerebral hemorrhage. J Clin Neurosci. 2012;19:1096-1100. doi:10.1016/j. jocn.2011.11.020

22. Seo WK, Seok HY, Kim JH, et al. C-reactive protein is a predictor of early neurologic deterioration in acute ischemic stroke. J Stroke Cerebrovasc Dis. 2012;21:181-186. doi:10.1016/j. jstrokecerebrovasdis.2010.06.002

23. Stavem K, Lossius M, Rønning OM. Reliability and validity of the Canadian neurological scale in retrospective assessment of initial stroke severity. Cerebrovasc Dis. 2003;16(3):286-291. doi:10.1159/ 000071129
24. Kimura Y, Yanagida T, Onda A, et al. Soluble uric acid promotes atherosclerosis via ampk (amp-activated protein kinase)-mediated inflammation. Arterioscler Thromb Vasc Biol. 2020;40:570-582. doi:10.1161/ATVBAHA.119.313224

25. Ruggiero C, Cherubini A, Ble A, et al. Uric acid and inflammatory markers. Eur Heart J. 2006;27:1174-1181. doi:10.1093/eurheartj/ ehi879

26. Kurajoh M, Fukumoto S, Yoshida S, et al. Uric acid shown to contribute to increased oxidative stress level independent of xanthine oxidoreductase activity in medcity 21 health examination registry. Sci Rep. 2021;11:7378. doi:10.1038/s41598-021-86962-0

27. Yao Z, Bai Q, Wang G. Mechanisms of oxidative stress and therapeutic targets following intracerebral hemorrhage. Oxid Med Cell Longev. 2021;2021:8815441. doi:10.1155/2021/8815441

28. Andalib S, Lattanzi S, Di Napoli M, et al. Blood pressure variability: a new predicting factor for clinical outcomes of intracerebral hemorrhage. J Stroke Cerebrovasc Dis. 2020;29(12):105340. doi:10.1016/j.jstrokecerebrovasdis.2020.105340

29. Divani AA, Liu X, Di Napoli M, et al. Blood pressure variability predicts poor in-hospital outcome in spontaneous intracerebral hemorrhage. Stroke. 2019;50(8):2023-2029. doi:10.1161/ STROKEAHA.119.025514
Neuropsychiatric Disease and Treatment

\section{Publish your work in this journal}

Neuropsychiatric Disease and Treatment is an international, peerreviewed journal of clinical therapeutics and pharmacology focusing on concise rapid reporting of clinical or pre-clinical studies on a range of neuropsychiatric and neurological disorders. This journal is indexed on PubMed Central, the 'PsycINFO' database and CAS, and is the official journal of The International Neuropsychiatric Association (INA). The manuscript management system is completely online and includes a very quick and fair peer-review system, which is all easy to use. Visit http://www.dovepress.com/testimonials.php to read real quotes from published authors. 